\title{
COMPLEMENTED MODULAR LATTICES AND PRO JECTIVE SPACES OF INFINITE DIMENSION
}

\author{
BY \\ ORRIN FRINK, JR.

\section{INTRODUCTION}

Garrett Birkhoff $[1]\left(^{1}\right)$ has shown that every complemented modular lattice of finite dimension is the direct union of lattices associated with projective geometries of finite dimension. The present paper is an attempt to generalize this characterization to the case of complemented modular lattices and projective spaces in general, with no restriction on the dimensionality.

There is one respect in which the infinite-dimensional case is more complicated than the finite-dimensional case. The general projective spaces we consider are atomic; they contain points or atoms. Consequently the lattices associated with them are atomic. But complemented modular lattices need not be atomic, as is shown by the example of continuous geometries. Our procedure is to show that every complemented modular lattice determines a complete atomic complemented modular lattice in which it is imbedded. This extension to an atomic lattice is accomplished by the use of maximal dual ideals. The resulting atomic lattice is then shown to be the direct union of irreducible projective spaces of a particular kind. The final characterization theorem we obtain states that every complemented modular lattice is the subdirect union of projective planes and irreducible projective coordinate spaces. A projective coordinate space is determined by an arbitrary cardinal number (its dimension) and an arbitrary division ring.

This result is not as neat as Birkhoff's characterization theorem, since it involves subdirect unions instead of direct unions. On the other hand, the preliminary step of imbedding the original lattice in an atomic lattice (a step which is not needed for finite-dimensional lattices, which are atomic) promises to be of use in connection with the theory of continuous geometries. Continuous geometries have no points, lines, or planes. It seems to have been assumed further that it is not possible to introduce points, lines, and planes into a continuous geometry in a reasonable way. Such an assumption would be incorrect. The introduction is not only possible, but it has the advantage that it allows one to associate a division ring with the continuous geometry by the method of von Staudt. This should lead to a simpler coordinatization of continuous geometries than the coordinatization by regular rings used by von

Presented to the Society, August 22, 1946; received by the editors March 29, 1946.

(1) Numbers in brackets refer to the bibliography at the end of the paper. 
Neumann [14]. I have not yet been able to show, however, that the atomic lattice determined by a continuous geometry is irreducible, or that the division ring associated with a continuous geometry is unique.

The present paper is divided into two parts. Part I deals with general projective spaces. These result from merely omitting some of the postulates for projective geometries given by Veblen and Young. In particular the postulate which restricts the dimensionality is omitted. It is remarkable how little difference the omissions make. The lattice of subspaces of a projective space is still complete, atomic, complemented, and modular, even in the infinitedimensional case. The von Staudt coordinatization goes through also in the general case.

Part II deals with complemented modular lattices, and in particular with the theory of lattice ideals. The principal results are the imbedding theorem, and the theorem that with the obvious definition of collinearity, the maximal dual ideals of a complemented modular lattice are the points of a projective space.

\section{Part I. Projective spaces}

By a projective space we shall mean any system of elements called points and of sets of points called lines which satisfy the following two postulates A and $B$ due to Veblen and Young [13]. (Points which are on the same line are said to be collinear.)

\section{Postulate A. Two distinct points are on one and only one line.}

Postulate B. If the points $P, Q$, and $R$ are not collinear, while the points $P, Q, X$ and $Q, R, Y$ are collinear, and if $X$ and $Y$ are distinct, then there is $a$ point $Z$ such that $P, R, Z$ and $X, Y, Z$ are each collinear.

Postulate A states that two distinct points determine a line, while postulate $\mathrm{B}$ states that two distinct lines of a plane determine a point.

Definitions. If a line contains only two points, it will be called degenerate. If a projective space contains any degenerate lines it will be called reducible; otherwise it will be called irreducible. Given any collection $K$ of mutually disjoint projective spaces, a new projective space called their direct union may be defined as follows: The points of the direct union $D$ are all the points of the different spaces that make up the collection; the lines of $D$ consist of all the lines of these spaces, and in addition all degenerate lines which can be formed by taking two points, one from each of two distinct, and hence disjoint, spaces of the collection $K$. It is easily verified that $D$ satisfies the postuates $A$ and $B$, and is therefore a projective space.

1. Decomposition into irreducible spaces. The following theorem is due to Garrett Birkhoff [2] and was proved also by Karl Menger [10].

THEOREM 1. Every projective space is either irreducible, or is the direct union, in a unique way, of irreducible projective spaces. 
Proof. Let us call two distinct points $P$ and $Q$ of the projective space $S$ equivalent, and write $P \sim Q$, if the line $P Q$ is not degenerate (that is, if it contains at least three points). It is easily seen from postulates $A$ and $B$ that this relation of equivalence is symmetric, reflexive, and transitive. Hence it determines a partition of the space $S$ into disjoint equivalence classes. It is then easy to verify that these equivalence classes are irreducible projective spaces, whose direct union is $S$, unless $S$ itself is irreducible, in which case there is just one equivalence class. Conversely, if $S$ is the direct union of irreducible projective spaces, then two points of $S$ are equivalent if and only if they are in the same irreducible space. Hence the decomposition of $S$ into irreducible spaces is unique. These irreducible spaces are called components of $S$.

2. The lattice of subspaces. With each projective space we now associate a lattice in the usual way.

Definition. A set of elements of a projective space $S$ is called a subspace of $S$ if it contains all points collinear with any two of its points. It follows that any subspace of a projective space is itself a projective space. In particular a set consisting of a single point is always a subspace.

THEOREM 2. The collection of all subspaces of a projective space is a complete lattice, if the lattice order relation is taken to be the relation of set inclusion between subspaces, and the operation of lattice meet is taken to be the operation of set intersection.

Proof. It is sufficient to show that the set intersection, or common part, of any collection, finite or infinite, of subspaces of a projective space is itself a subspace. But this follows immediately from the definition of subspace.

3. The join theorem. The lattice meet of two subspaces is their intersection. It will now be shown that the lattice join of two subspaces is their join in the sense of projective geometry.

THEORFM 3. If $A$ and $B$ are two distinct and non-empty subspaces of a projective space $S$, then the lattice join $A \cup B$ in the lattice of all subspaces of $S$ consists of all points on lines of $S$ which contain two distinct points, one from $A$ and one from $B$.

Proof. Consider first the case where $S$ is irreducible. The join $A \cup B$ is the smallest subspace of $S$ containing both $A$ and $B$. Hence $A \cup B$ necessarily includes the set $J$ consisting of all points on lines joining points of $A$ to different points of $B$. If $J$ is itself a subspace, it must coincide with $A \cup B$, which is to be proved. Hence we must show that $J$ contains all points collinear with two of its points. Let $C_{3}$ be any point of $S$ collinear with two points $C_{1}$ and $C_{2}$ of $J$. Then $C_{1}$ is collinear with points $A_{1}$ and $B_{1}$ of $A$ and $B$, and $C_{2}$ is collinear with points $A_{2}$ and $B_{2}$ of $A$ and $B$. It must be shown that $C_{3}$ is collinear with points $A_{3}$ and $B_{3}$ of $A$ and $B$. If the four points $A_{1}, B_{1}, A_{2}, B_{2}$ are coplanar (that is, if they lie in the subspace determined by three of them), it follows 
directly from postulate $\mathrm{B}$ that either line $A_{1} C_{3}$ or line $B_{1} C_{3}$ contains points of both $A$ and $B$.

Otherwise the four points $A_{1}, B_{1}, A_{2}, B_{2}$ determine an ordinary projective three-space $S_{3}$, and the conclusion follows from well known properties of such a three-space (for instance, see Veblen and Young, Projective geometry, vol. 1, pp. 20-25). For the planes $A_{1} A_{2} C_{3}$ and $B_{1} B_{2} C_{3}$ of $S_{3}$ intersect in a line $L$ which contains the point $C_{3}$ and which meets the line $A_{1} A_{2}$ in a point $A_{3}$ and which also meets the line $B_{1} B_{2}$ in a point $B_{3}$. Hence $C_{3}$ is collinear with points $A_{3}$ and $B_{3}$ of $A$ and $B$ respectively, which was to be proved.

The case where the space $S$ is reducible can be treated in a similar way. If the seven given points $A_{1}, B_{1}, C_{1}, A_{2}, B_{2}, C_{2}$, and $C_{3}$ are distinct, it follows from the transitivity of the equivalence relation that they all lie in the same component of $S$, and the proof is as before. On the other hand, if there are any coincidences among the seven points, the conclusion follows directly from postulate $\mathrm{B}$. In other words, this case can be handled by plane geometry, without the use of theorems about three-space. This completes the proof.

4. The second join theorem. The join of an infinite number of subspaces has also a simple characterization.

Theorem 4. In the lattice of subspaces of a projective space $S$, the join of an infinite collection $K$ of subspaces of $S$ consists of all points which belong to the. joins of finite subcollections of $K$.

Proof. The set of points described in the statement of the theorem is a subspace of $S$, since a point collinear with points of two finite joins $F$ and $F^{\prime}$ is a point of their join $F \cup F^{\prime}$, which is itself a finite join. It is clearly the smallest subspace which includes all subspaces of the collection $K$, and is therefore the join of $K$.

5. The lattice of subspaces is complemented and modular. To show that the lattice of subspaces is complemented requires the use of Zorn's lemma, although modularity is proved as in the finite-dimensional case.

Theorem 5. The lattice $L$ of all subspaces of a projective space $S$ is complemented and modular.

Proof. To prove modularity, it must be shown that $(X \cup Y) \cap Z \leqq X$ $\cup(Y \cap Z)$ provided $X \leqq Z$, where $X, Y$, and $Z$ are subspaces of $S$. Let $u$ be any point of $(X \cup Y) \cap Z$; then $u$ is in $X \cup Y$ and also in $Z$. By the join theorem, $u$ is collinear with points $x$ and $y$ of $X$ and $Y$. Now $u$ is in $Z$, and $x$ is also in $Z$ since $X \leqq Z$. Hence $y$, being collinear with $x$ and $u$, is also in $Z$, since $Z$ is a subspace. Therefore $y$ is in $Y \cap Z$. Consequently $u$ is collinear with a point $x$ of $X$ and a point $y$ of $Y \cap Z$. Hence $u$ is in $X \cup(Y \cap Z)$, which was to be proved.

We now show that the lattice $L$ of subspaces of $S$ is complemented. Let $A$ be any subspace of $S$. If $A$ is the entire space $S$, then it has a complement, 
namely the empty subspace of $S$. Otherwise there are points, and hence nonempty subspaces of $S$, which are disjoint from $A$. Consider the collection $K$ of all subspaces of $S$ which have no point in common with $A$. By Zorn's lemma, the collection $K$ contains a maximal subspace $B$. For if $H$ is any subcollection of $K$ the members of which are simply ordered by set inclusion, then the set union of all sets of $H$ is also in $K$, since it is disjoint from $A$ and is also a subspace of $S$. We shall show that this maximal element $B$ is a complement of $A$.

It is clear that $A \cap B=O$, since $B$ is in $K$. To prove that $A$ and $B$ are complements, it must also be shown that $A \cup B=S$. However, if $A \cup B$ were distinct from $S$, there would exist a point $P$ of $S$ not in $A \cup B$. Then $B \cup P$ would be disjoint from $A$, and $B$ would not be maximal in $K$. For if $B \cup P$ contained a point $Q$ of $A$, then by the join theorem $Q$ would be collinear with $P$ and a point $R$ of $B$. Then $P$ would be collinear with points $Q$ of $A$ and $R$ of $B$, hence $P$ would be in the subspace $A \cup B$, contrary to assumption. This contradiction completes the proof.

6. Projective coordinate spaces. We shall now show how to construct an irreducible projective space $P S(\alpha, D)$ of arbitrary dimension $\alpha$, with coordinates from an arbitrary division ring $D$.

Let $\alpha$ be any cardinal number, let $T$ be a set of elements of cardinal number $\alpha$, and let $t$ be a variable ranging over the set $T$. Let $D$ be any division ring. We shall call a function $d(t)$ a coordinate function if :

(1) the function $d(t)$ is defined over the set $T$.

(2) The functional values of $d(t)$ are in the ring $D$.

(3) The function $d(t)$ is not identically zero, but $d(t)=0$ except for a finite set of values of $i$.

Here the symbol 0 stands for the zero element of the ring $D$.

If $d(t)$ is a coordinate function, and $r$ is any nonzero element of the ring $D$, then $r d(t)$, obtained by multiplying all the functional values of $d(t)$ on the left by $r$, is also a coordinate function.

If $d(t)$ is a coordinate function, then we shall define the point $P$ of the space $P S(\alpha, D)$ belonging to the function $d(t)$ to be the set of all coordinate functions $r d(t)$, where $r$ is in $D$ and $r \neq 0$. Since $D$ is a ring, it is clear that $P$ belongs likewise to all the other coordinate functions of the set $P$, and that $P$ is determined by any one of its coordinate functions.

Three points $P, P^{\prime}, P^{\prime \prime}$ of $P S(\alpha, D)$ will be called collinear if they have coordinate functions $d(t), d^{\prime}(t), d^{\prime \prime}(t)$ respectively such that $d(t)+d^{\prime}(t)+d^{\prime \prime}(t)$ $=0$.

Theorem 6. The space PS $(\alpha, D)$ is an irreducible projective space.

Proof. If we define a line to be the set of all points collinear with two distinct points, it is easy to see that postulate $A$ for projective spaces is satisfied. To show that postulate $\mathrm{B}$ is satisfied, let $P, P^{\prime}, P^{\prime \prime}$ be three noncollinear 
points with coordinate functions $d(t), d^{\prime}(t), d^{\prime \prime}(t)$ respectively, and let $Q$ with coordinate function $r d(t)+r^{\prime} d^{\prime}(t)$ be on line $P P^{\prime}$, and $Q^{\prime}$ with coordinate function $r^{\prime} d^{\prime}(t)+r^{\prime \prime} d^{\prime \prime}(t)$ be on line $P^{\prime} P^{\prime \prime}$. If $Q$ and $Q^{\prime}$ are distinct, $r$ and $r^{\prime \prime}$ are not both zero. Then $r d(t)-r^{\prime \prime} d^{\prime \prime}(t)$ is not identically zero, and is therefore the coordinate function of a point $Q^{\prime \prime}$ which is on line $P P^{\prime \prime}$ and also on line $Q Q^{\prime}$. Hence postulate $B$ is satisfied.

The projective space $P S(\alpha, D)$ is irreducible, since the line joining the distinct points $P$ and $P^{\prime}$ with coordinate functions $d(t)$ and $d^{\prime}(t)$ always contains at least a third point $P^{\prime \prime}$ with coordinate function $d(t)-d^{\prime}(t)$.

7. Coordinatization of projective spaces. It has been shown in Theorem 6 that every projective coordinate space $P S(\alpha, D)$ is an irreducible projective space. We now consider a sort of converse of this theorem. Is every irreducible projective space a coordinate space $P S(\alpha, D)$ ? Obviously not, since it is well known that there exist projective planes which are peculiar and cannot be coordinatized by rings [9]. With this single exception, however, a converse of Theorem 6 can be proved. The proof is complicated, since it involves the introduction of a coordinate system into an infinite-dimensional space.

THEOREM 7. Every irreducible projective space is either a projective plane or is isomorphic to a projective coordinate space $P S(\alpha, D)$.

Proof. Suppose $S$ is an irreducible projective space which is not a projective plane. We shall now define what is meant by an independent set of points of $S$. A finite set of points of $S$ is said to be independent if no point of the set is in the join of the other points of the set. An infinite set of points $I$ of $S$ is said to be independent if every finite subset of $I$ is independent. Referring to the second join theorem (Theorem 4) it is seen that a set of points, finite or infinite, is independent if and only if it is true that no point of the set is in the join of the other points of the set.

In order to construct a coordinate system in $S$, we need to have a maximal independent set of points of $S$, to serve as the vertices of a coordinate simplex. It follows from Zorn's lemma that such a maximal independent set of points of $S$ exists. For consider the collection $K$ of all independent sets of $S$. The set union of any linearly ordered subcollection $H$ of the collection $K$ is clearly an independent set, and is therefore in $K$. The hypothesis of Zorn's lemma is verified, hence the conclusion holds, namely, that there exists a maximal set $T$ in $K$, that is, a maximal independent set $T$ of points of $S$.

Let $\alpha$ be the cardinal number of the set $T$. We shall call $\alpha$ the projective dimension of the space $S$. If $\alpha$ is finite, it is one greater than the ordinary dimension of $S$.

Select a particular point $t_{0}$ of $T$. The set complement $T-t_{0}$ has a join $H_{0}$ in the lattice of subspaces of $S$. It is easily verified that $H_{0}$ is a complement of the subspace $t_{0}$ in this lattice. Furthermore $H_{0}$ is a maximal element of the lattice, and is called a hyperplane. 
Since the points of $T$ are to be the vertices of a coordinate simplex, we wish the lines $t_{0} \cup t_{a}$ joining $t_{0}$ to the other points $t_{a}$ of $T$ to be coordinate axes. Hence it is necessary to set up a scale on each such line. We first select arbitrarily on each line $t_{0} \cup t_{a}$ a third point $t_{a}^{\prime}$ distinct from $t_{0}$ and $t_{a}$. This is possible since the space $S$ is irreducible. Call $t_{a}^{\prime}$ the unit point of line $t_{0} \cup t_{a}$. Let $t_{1}$ be a particular point of $T$ distinct from $t_{0}$. The plan is to set up a scale on line $t_{0} \cup t_{1}$, and then to project this scale onto the lines $t_{0} \cup t_{a}$ so that unit points correspond, and so that the point $t_{1}$ corresponds to point $t_{a}$.

Let $t_{1}^{\prime}$ be the unit point of line $t_{0} \cup t_{a}$. We may now define a division ring $D$ whose elements are all the points of the line $t_{0} \cup t_{1}$ except point $t_{1}$, in such a way that $t_{0}$ is the zero element of $D, t_{1}^{\prime}$ is the unit element of $D$, while $t_{1}$ is the "infinite point" of the line. This can be done by the method of von Staudt (see Veblen and Young, Projective geometry, vol. 1, pp. 141-167) provided $S$ contains a projective three-space $S_{3}$ containing the line $t_{0} \cup t_{1}$. Except in the trivial cases where $S$ consists of a single line or of a single point, $S$ will contain such a three-space, since by assumption $S$ is irreducible and is not a projective plane.

This division ring is the ring $D$ of the space $P S(\alpha, D)$ we are to construct. It determines a coordinate system on the line $t_{0} \cup t_{1}$, whereby to each point $P$ of the line $t_{0} \cup t_{1}$ other than $t_{1}$ there is assigned as coordinate the point $P$ itself, considered as an element of the ring $D$.

We now set up also on each other coordinate axis $t_{0} \cup t_{a}$ a scale or coordinate system by means of a perspectivity projecting the points of $t_{0} \cup t_{1}$, and hence the elements of the ring $D$, onto the points of the line $t_{0} \cup t_{a}$, so that the point $t_{1}$ goes into the point $t_{a}$, while the unit point $t_{1}^{\prime}$ goes into the unit point $t_{a}^{\prime}$. This perspectivity has its center at the point $\left(t_{1} \cup t_{a}\right) \cap\left(t_{1}^{\prime} \cup t_{a}^{\prime}\right)$, which is in the hyperplane $H_{0}$.

Now let $P$ be any point of $S$ not in $H_{0}$. By the second join theorem, $P$ is in the join of a finite independent subset $T_{n}$ of points of $T$ which we shall call the points $t_{0}, t_{1}, \cdots, t_{n}$. We wish to assign a coordinate function $d(t)$ to the point $P$, where $t$ ranges over the set $T$, and the function $d(t)$ takes values in the ring $D$. We define $d(t)$ to be zero if $t$ is not one of the points $t_{0}, \cdots, t_{n}$. We define $d\left(t_{0}\right)$ to be 1 , the unit element of the ring $D$. If $i$ is one of the subscripts $1,2, \cdots, n$, we define $d\left(t_{i}\right)$ to be the coordinate on $t_{0} \cup t_{i}$ of the point

$$
\left(t_{1} \cup t_{2} \cup \ldots \cup t_{i-1} \cup P \cup t_{i+1} \cup \ldots \cup t_{n}\right) \cap\left(t_{0} \cup t_{i}\right)
$$

In other words, we assign to each point in the finite-dimensional projective space determined by the finite set $T_{n}$ a set of coordinates in the usual way.

On the other hand, if $P$ is any point of the hyperplane $H_{0}$, then $P$ is in the join of a finite independent set of points $t_{1}, t_{2}, \cdots, t_{n}$ of $T$ other than $t_{0}$. Let $Q$ be any point of the line $t_{0} \cup P$ other than $t_{0}$ or $P$. Since $Q$ is not in $H_{0}$, a coordinate function $d(t)$ has already been assigned to it. In terms of this coordinate function $d(t)$ of the point $Q$, we define a coordinate function $d^{\prime}(t)$ for the point $P$ so that $d^{\prime}\left(t_{0}\right)=0$, while $d^{\prime}(t)=d(t)$ if $t$ is distinct from $t_{0}$. 
In this way every point of $S$ has a coordinate function assigned to it. By multiplying each such coordinate function $d(t)$ on the left by all nonzero elements $r$ of the ring $D$, we get a set of coordinate functions of the form $r d(t)$, which set is by definition a point of the projective coordinate space $P S(\alpha, D)$. Thus each point of the space $S$ has been set into correspondence with a point of the space $P S(\alpha, D)$. It remains to show that this correspondence between projective spaces is an isomorphism.

To show this, let $P, Q$, and $R$ be three distinct points of the space $S$. They lie in the join $J$ of a finite number of points $t_{1}, t_{2}, \cdots, t_{n}$ of the maximal independent set $T$. This subspace $J$ is an ordinary projective space of finite projective dimension $n$, and our coordinate functions were assigned to points of $J$ in the same manner that ordinary projective coordinates would be assigned to these points, considered as points of $J$ rather than of $S$. Hence the condition, in terms of coordinates, that the three points $P, Q$, and $R$ be collinear in $S$ is the same as the condition that the corresponding points of $P S(\alpha, D)$ be collinear. Likewise distinct points of $J$ have linearly independent coordinates in $J$, and hence have disjoint sets of coordinate functions assigned to them in $P S(\alpha, D)$.

This shows that the spaces $S$ and $P S(\alpha, D)$ are isomorphic, and completes the proof of Theorem 7 .

CoROLlARY. Every projective space is either a projective plane, or a projective coordinate space $P S(\alpha, D)$, or a direct union of spaces of these two types.

\section{PaRT II. Ideals IN COMPLEMENTED MODUlaR LATTICES}

It has already been shown (Theorem 5) that the lattice of subspaces of a projective space is complemented and modular. We now wish to show that every complemented modular lattice determines a projective space in whose lattice it is imbedded. To do so we shall first imbed the given complemented modular lattice in an atomic lattice by the use of ideal theory. We first derive some results concerning atomic lattices. In dealing with lattices we shall assume a familiarity with Garrett Birkhoff's book Lattice theory, and we shall make use of the notation and terminology of that book.

8. Atomic lattices. By an atom or point of a lattice with a zero element is meant a minimal nonzero element, that is, an element that covers the zero element. A complemented modular lattice will be called atomic if corresponding to every nonzero element $A$ of the lattice there exists at least one atom $P$ such that $P \leqq A$. By the representative set $r(A)$ of an element $A$ of a complemented modular atomic lattice $L$ is meant the set of all atoms $P$ of $L$ such that $P \leqq A$.

THEOREM 8. Every element $A$ of a complemented modular atomic lattice is the join of the elements (atoms) of its representative set $r(A)$.

Proof. If $A$ is the zero element of the lattice, its representative set is 
empty, and the join of the elements of this empty set is $A$, by the usual convention. Otherwise $A$ is an upper bound of the elements of $r(A)$. We must prove it is the least upper bound. Suppose it is not; let $B$ be an upper bound and $B<A$. Since the lattice is complemented and modular there exists a relative complement $C$ of $B$ in $A$; then $A=B \cup C, B \cap C=0, C \neq O$. Since the lattice is atomic, there is an atom $P$ such that $P \leqq C$. Then $P \leqq A$, since $C \leqq A$. Hence $P \in r(A)$, and $P \leqq B$, since $B$ is an upper bound of the elements of $r(A)$. But this is impossible since $B \cap C=0$. This completes the proof.

9. The representation of complemented modular atomic lattices by sets. The following theorem generalizes some well known results on Boolean algebras.

THEOREM 9. The representative sets of a complemented modular atomic lattice $L$ form a lattice isomorphic to $L$, with set inclusion as the lattice order relation, and set intersection as the operation of lattice meet.

Proof. We first show that $r(A \cap B)=r(A) \wedge r(B)$, where the symbol $\wedge$ denotes set intersection (or logical product). If the atom $P$ is a member of $r(A \cap B)$, then by definition $P \leqq A \cap B$. Hence $P \leqq A$ and $P \leqq B$; it follows that $P \in r(A)$ and $P \in r(B)$, and hence $P \in[r(A) \wedge r(B)]$. Conversely if $P \in[r(A) \wedge r(B)]$, then $P \leqq A$ and $P \leqq B$; hence $P \leqq A \cap B$ and $P \in r(A \cap B)$.

We next show that $r(A \cup B)$ is the least upper bound relative to set inclusion of $r(A)$ and $r(B)$ in the collection of representative sets. This will prove that the collection is a lattice, homomorphic to $L$. Suppose then that $r(A \cup B)$ is not least upper bound of $r(A)$ and $r(B)$ in the system of representative sets. Then there exists a representative set $r(C)$ such that $r(A) \subset r(C)$ and $r(B) \subset r(C)$, but such that $r(A \cup B) \subset r(C)$ is false. Hence there is an atom $P$ such that $P \leqq A \cup B$ is true, but $P \leqq C$ is false. But $r(C)$ contains all atoms of $r(A)$ and $r(B)$, since it is an upper bound of these sets; hence $A \leqq C$ and $B \leqq C$ since by Theorem $8, A$ and $B$ are respectively the joins of all elements of $r(A)$ and $r(B)$. Hence $C \geqq A \cup B$ and $P \leqq C$ since $P \leqq A \cup B$, contrary to assumption.

This shows that the lattice $L$ and the lattice of all its representative sets are homomorphic. It remains to show that they are isomorphic. Suppose that $A$ and $B$ are distinct elements of $L$; it must be shown that $r(A)$ and $r(B)$ are distinct. It follows from Theorem 8 that $A$ is the join of all elements of $r(A)$, and $B$ is the join of all elements of $r(B)$. Hence if $r(A)$ and $r(B)$ were identical, then $A$ would be identical with $B$. This contradiction completes the proof.

10. The lattice of dual ideals. By a dual ideal $I$ of a lattice $L$ is meant a non-empty set of elements of $L$ such that the lattice meet $A \cap B$ of two elements of $L$ is in $I$ if and only if both $A$ and $B$ are in $I$. A dual ideal $I$ is said to be maximal if it is not the entire lattice and if the only dual ideal with more elements than $I$ is the entire lattice. A dual ideal $I$ not the entire lattice is said to be prime if whenever the lattice join $A \cup B$ is in $I$, then either $A$ or $B$ is in $I$. Ideals in modular lattices have already been studied by Garrett 
Birkhoff, Dilworth, Gorn, and others $[1,5,8]$. Ideals in Boolean algebras and in distributive lattices have been studied by M. H. Stone $[11,12]$. It is interesting to note that in Boolean algebras the notions of maximal ideal and prime ideal coincide, while in merely distributive or modular lattices they do not. In distributive lattices it is the prime ideals that are most important, while in complemented modular lattices the maximal ideals are of greater importance, since prime ideals may not even exist.

Theorem 10. The collection $D(L)$ of all dual ideals of a lattice $L$ is a complete lattice which is modular when $L$ is modular, provided the operation of lattice join $I \cup J$ in $D(L)$ is taken to mean set intersection, and the relation of lattice inclusion $I \leqq J$ is taken to be the superset relation $I \supset J$.

Proof. To show that $D(L)$ is a complete lattice, it is sufficient to verify that the set intersection of any collection of dual ideals is again a dual ideal. That $D(L)$ is modular when $L$ is modular was proved by Dilworth [5]. The reversal of what might seem the more natural lattice order relation in $D(L)$ is merely a matter of convenience and not of necessity. It is adopted also by Dilworth. No originality is claimed for Theorem 10, which is listed as a theorem as a matter of convenience.

11. Principal dual ideals. If $A$ is an element of a lattice $L$, then the set of all elements $B$ of $L$ such that $A \leqq B$ is a dual ideal which is called a principal dual ideal, and is denoted by $[A]$.

THEOREM 11. The collection of all principal dual ideals of a lattice $L$ is a sublattice of the lattice of all dual ideals of $L$, and is isomorphic to $L$.

The proof of this theorem will be omitted, since it follows well known lines and does not involve anything essentially new.

12. The extension of a dual ideal to be maximal. The following theorem is a generalization of a result of $\mathrm{M}$. $\mathrm{H}$. Stone concerning ideals in Boolean algebras $[7,11]$.

THEOREM 12. If $L$ is a lattice with a zero element, then any dual ideal I of $L$ which is neither maximal nor identical with $L$ can be extended to be maximal.

Proof. We use Zorn's lemma. Since $I$ is not the entire lattice, $I$ does not contain the zero element of $L$. Let $K$ be the collection of all dual ideals $J$ of $L$ such that (1) $I$ is a subset of $J$, and (2) $J$ does not contain the zero element. The union of any subcollection of ideals of $K$ which is linearly ordered by set inclusion is clearly an ideal which satisfies (1) and (2) and is therefore in $K$. Hence the hypothesis of Zorn's lemma is satisfied, and the conclusion follows, namely that $K$ has a maximal element. This maximal element is a maximal dual ideal containing $I$ as a subset, whose existence was to be proved.

COROLlary. The lattice of all dual ideals of a complemented modular lattice is complete, atomic, and modular. 
This follows from Theorems 10 and 12. In particular, it follows from Theorem 12 that the lattice of all dual ideals is atomic, since in this lattice maximal dual ideals are atoms, and every element can be extended to be maximal, therefore every element includes an atom. Unfortunately, the lattice of all dual ideals of a complemented modular lattice is not necessarily complemented. For example, let $L$ be a continuous geometry. Then $L$ has no atoms (points), likewise no maximal elements (hyperplanes). The lattice $D(L)$ of all dual ideals of $L$ has atoms (namely maximal dual ideals), but these atoms have no complements in $D(L)$. For, such a complement would be a minimal dual ideal. These do not exist, for if $I$ were a minimal dual ideal, and $A$ an element of $I$, then the principal dual ideal $[A]$ would be a subset of $I$. Since $I$ is minimal, it would coincide with $[A]$. Hence the element $A$ would be maximal in $L$; but $L$ has no maximal elements.

To be sure, one could consider the dual concept of the lattice $D(L)$, namely the lattice of all ideals (multiplicative ideals) instead of dual ideals. Among these would be found maximal ideals, which would serve as complements of maximal dual ideals, if both kinds of ideal could be combined in a single lattice.

In this paper a simpler procedure will be followed in order to imbed a complemented modular lattice $L$ in a larger complemented modular lattice which is also complete and atomic. It will be shown that the maximal dual ideals of $L$ constitute the points of a projective space, when the notion of collinearity is defined in a suitable way. The subspace lattice of this projective space is then complete, atomic, complemented, and modular, and contains a sublattice isomorphic to $L$.

13. Collinearity and the projective space of maximal dual ideals. If $P$ and $Q$ are two distinct maximal dual ideals of a complemented modular lattice $L$, then the join (that is, the set intersection) $P \cup Q$ of $P$ and $Q$ in the lattice of all dual ideals of $L$ will be called the linear ideal determined by $P$ and $Q$. By the line $P Q$ determined by $P$ and $Q$ will be meant the set of all maximal dual ideals $R$ of $L$ such that $R \leqq P \cup Q$. Maximal dual ideals will be called collinear if they are on the same line.

Note the important distinction between the linear ideal $P \cup Q$ and the line $P Q$. The former is a dual ideal; the latter is a set of dual ideals.

Theorem 13. The maximal dual ideals of any complemented lattice are the points of a projective space.

Proof. It must be shown that postulates A and B for projective spaces are satisfied for the system whose points are all the maximal dual ideals of a complemented modular lattice $L$, if the notion of line is defined as above.

Postulate A states that two distinct points are on one and only one line. It is clear from the definition of line that two distinct maximal dual ideals $P$ and $Q$ are on at least one line, namely the line $P Q$. Suppose now that $R$ is a 
point of the line $P Q$ distinct from the points $P$ and $Q$. We wish to show that the line $P R$ is identical with the line $P Q$. This follows from the fact that the lattice $D(L)$ of dual ideals is modular (Theorem 10). Consider the linear ideals $P \cup Q$ and $P \cup R$. By hypothesis $R \leqq P \cup Q$, hence $P \cup R \leqq P \cup Q$. But $P$ and $Q$, being atoms, cover the zero element of $D(L)$. Since the lattice is modular, $P \cup Q$ covers $P$. Since $P \leqq P \cup R \leqq P \cup Q$, and $P$ and $R$ are distinct, it follows that $P \cup Q=P \cup R$, and hence lines $P Q$ and $P R$ are identical. It follows that any two points of a line determine the line, and hence postulate $A$ is satisfied.

The proof that postulate $B$ is satisfied is based on the fact that, in a modular lattice, if the elements $X$ and $Y$ are distinct and both of them cover the element $A$, then the element $X \cup Y$ covers $X$ and $Y$; while if $A$ covers $X$ and $Y$, then $X$ and $Y$ cover $X \cap Y$ (Garrett Birkhoff, Lattice theory, p. 34).

Suppose the hypothesis of postulate B holds; then $P, Q, R$ are not collinear, but $P, Q, X$ and $Q, R, Y$ are collinear, and $X$ and $Y$ are distinct. It must be shown that lines $P R$ and $X Y$ intersect in a point. This is equivalent to showing that the element $(P \cup R) \cap(X \cup Y)$ of the lattice $D(L)$ is not the zero element. Now it follows from the covering properties of modular lattices just described that we have in turn:

(1) $P \cup Q$ covers $X$,

(2) $Q \cup R$ covers $Y$,

(3) $P \cup Q \cup R$ covers $P \cup R$,

(4) $P \cup Q \cup R$ covers $X \cup Y$,

(5) $P \cup R$ covers $(P \cup R) \cap(X \cup Y)$.

It follows from (5) that $(P \cup R) \cap(X \cup Y)$ is not the zero element, since it is covered by $P \cup R$, which in turn covers the elements $P$ and $R$, which are not zero. This is assuming the elements $P$ and $R$ are distinct. In the contrary case the conclusion follows easily. This completes the proof of Theorem 13.

14. Representative sets and the imbedding theorem. It has just been shown that every complemented modular lattice determines a projective space. By Theorem 5 the lattice of all subspaces of this projective space is complete, atomic, complemented, and modular. We now wish to show that the original lattice is imbedded in this lattice of subspaces.

If $A$ is any element of the complemented modular lattice $L$, then by the representative set $r(A)$ of $A$ will be meant the set of all maximal dual ideals $P$ of $L$ such that $A$ is an element of $P$. This is related to the notion of representative set defined in (8) for atomic lattices. In fact, if we pass from the original lattice $L$ to the lattice $D(L)$ of all dual ideals, which is atomic with maximal dual ideals for atoms, then the representative set of an element $A$ of $L$ according to the definition just given is identical with the representative set of the principal dual ideal $[A]$ of $D(L)$ according to the definition of (8). Note that by Theorem 11 the lattice of all principal dual ideals of $L$ is isomorphic to $L$.

It might be thought that the desired result would now follow from Theorem 9, which states that an atomic complemented modular lattice is iso- 
morphic to the lattice of its representative sets. Unfortunately the lattice $D(L)$, though atomic and modular, is not necessarily complemented, and Theorem 9 does not apply to it. We also wish to show not merely that the representative sets of elements of $L$ form a lattice isomorphic to $L$, but also that this lattice is a sublattice of the lattice of subspaces of the projective space determined by $L$.

Note that if $A$ is the zero element of $L$, its representative set is empty, while the representative set of the unit element $I$ of $L$ is the set of all maximal dual ideals.

THEOREM 14. The representative sets of elements of a complemented modular lattice $L$ form a lattice isomorphic to $L$. This lattice is a sublattice of the lattice of all subspaces of the projective space $S$ determined by $L$.

Proof. Note that any representative set $r(A)$ is a subspace of the projective space $S$, the points of which are the maximal dual ideals of the lattice $L$. For if $P$ and $Q$ are any two distinct points (maximal dual ideals) of $r(A)$, then the linear ideal $P \cup Q$ is the set intersection of ideals $P$ and $Q$, and the lattice element $A$, since it is a member of both $P$ and $Q$, is also a member of $P \cup Q$. Hence if $R$ is any point collinear with $P$ and $Q$, then $P \cup Q$ is a subset of $R$. Hence $A$ is a member of $R$, and $R$ is a member of $r(A)$. There fore $r(A)$ is a subspace, since it contains all points collinear with two of its points.

Since representative sets are subspaces of the projective space $S$, the lattice operations of meet and join are defined for them. It will now be shown that the correspondence between elements $A$ of $L$ and their representative sets $r(A)$ preserves the lattice operations of meet and join. It can be seen that $r(A) \cap r(B)=r(A \cap B)$. For the maximal dual ideal $P$ is an element of the representative set $r(A \cap B)$ if and only if $P$ is an element of both $r(A)$ and $r(B)$, since by definition of representative set, this is equivalent to saying that $A \cap B$ is a member of $P$ if and only if both $A$ and $B$ are members of $P$, which follows from the definition of dual ideal. This is because the lattice meet of the subspaces $r(A)$ and $r(B)$ is their intersection.

To show that the correspondence preserves the operation of lattice join is more difficult. First note that $r(A) \cup r(B) \leqq r(A \cup B)$. For if $P$ is a point of $r(A) \cup r(B)$, then by the join theorem (Theorem 3) $P$ is collinear with points $Q$ and $R$ of $r(A)$ and $r(B)$ respectively. Hence the linear ideal $Q \cup R$ is a subset of $P$. It follows that $A \cup B$ is an element of $P$, and $P$ is a member of $r(A \cup B)$.

Next it must be shown that $r(A \cup B) \leqq r(A) \cup r(B)$. This means, according to the join theorem, that if the point $P$ is a member of the representative set $r(A \cup B)$, then $P$ is collinear with points $Q$ and $R$ of the representative sets $r(A)$ and $r(B)$ respectively. It may be assumed that neither $A$ nor $B$ is a member of $P$, for in that case the conclusion follows. We may further assume that $A \cap B=O$, since otherwise $A$ may be replaced by the relative complement $A^{\prime}$ of $B$ in $A \cup B$. 
Since $P$, as well as the points $Q$ and $R$ which we seek, is a member of the lattice of all dual ideals $D(L)$, we now consider the principal dual ideals $[A]$, $[B]$, and $[A \cup B]$ which are also in $D(L)$, rather than the elements $A, B$, and $A \cup B$, which are in $L$. Since $P \leqq[A \cup B]$, it follows that the dual ideal $([B] \cup P) \cap[A]$ is not the zero element of $D(L)$, since otherwise both $[B]$ and $[B] \cup P$ would be distinct relative complements of $[A]$ in $[A \cup B]$, which is impossible in a modular lattice. For we have: $[A] \cup([B] \cup P)=[A \cup B]$, $[A] \cup[B]=[A \cup B]$, and $[A] \cap[B]=O$.

Hence by Theorem 12 the ideal $([B] \cup P) \cap[A]$ may be extended to be a maximal dual ideal $Q$. It follows that $A$ is a member of $Q$, and $Q \leqq[B] \cup P$. Consider now the ideal $(P \cup Q) \cap[B]$. If this were the zero element of $D(L)$, then both $P$ and $P \cup Q$ would be distinct relative complements of $[B]$ in $[B] \cup P$, since we would have $[B] \cup(P \cup Q)=[B] \cup P$, and $[B] \cap(P \cup Q)$ $=[B] \cap P=O$. But this is impossible in a modular lattice since $[B]$ and $[B] \cup P$ are distinct. Hence $[B] \cap(P \cup Q)$, since it is not zero, is a maximal dual ideal which we may call $R$, such that $R \leqq[B]$. Hence $R$ is a member of $r(B)$, and $P$ is collinear with points $Q$ and $R$ of $r(A)$ and $r(B)$ respectively, which was to be proved, since it can be seen that $P, Q$, and $R$ are collinear.

This completes the proof that the lattice operations correspond in the original lattice $L$ and in the lattice of representative sets. It remains to show that the correspondence is one-to-one and hence an isomorphism, or in other words that if $A$ and $B$ are distinct, then their representative sets $r(A)$ and $r(B)$ are distinct. Suppose $A$ and $B$ are distinct; then either $A \neq A \cap B$, or $B \neq A \cap B$. By symmetry we may suppose the former, that is, $A \neq A \cap B$. Since $L$ is complemented and modular, there exists in $L$ an element $A^{\prime}$ which is a relative complement of $A \cap B$ in $A$, that is, such that $A^{\prime} \cup(A \cap B)=A$, while $A^{\prime} \cap(A \cap B)=A^{\prime} \cap B=O$. This element $A^{\prime}$ is not the zero element of $L$, since otherwise $A=A^{\prime} \cup(A \cap B)=A \cap B$, contrary to assumption. Hence it follows from Theorem 12 that $A^{\prime}$ is an element of some maximal dual ideal $P$. Then $P$ is a member of $r(A)$, but not of $r(B)$, since otherwise $A^{\prime} \cap B=O$ would be an element of $P$, which is impossible since the zero element is never a member of a maximal dual ideal. It follows that $r(A)$ and $r(B)$ are distinct, which was to be proved. This completes the proof of Theorem 14 .

15. Characterization of complemented modular lattices. We have now shown that every complemented modular lattice determines a projective space (Theorem 13) in whose lattice of subspaces it is isomorphically imbedded (Theorem 14). Projective spaces have been characterized in the corollary to Theorem 7 as direct unions of projective planes and projective coordinate spaces $P S(\alpha, D)$. This leads to a characterization of complemented modular lattices as complemented sublattices of direct unions of the subspace lattices determined by projective planes and projective coordinate spaces. (It is easily verified that the subspace lattice of a projective space which is the direct union in the sense of this paper of irreducible projective spaces is 
isomorphic to the direct union, in the sense of abstract algebra or lattice theory, of the subspace lattices of those spaces.) This result is most conveniently described in terms of the notion of subdirect union due to Garrett Birkhoff [3]. An abstract algebra (in particular a lattice) $A$ is said to be a subdirect union of the collection $K$ of algebras (lattices) if $A$ is a subalgebra (sublattice) of the direct union of the members of $K$.

THEOREM 15. Every complemented modular lattice is a subdirect union of the subspace lattices of projective planes and irreducible projective coordinate spaces of type $P S(\alpha, D)$.

16. Conclusion. There are several questions that naturally suggest themselves in connection with these results. One of them is the following: if a complemented modular lattice is irreducible (not a direct union), is the projective space of maximal dual ideals determined by it also irreducible? If not, what additional conditions on the lattice are needed to insure the irreducibility of this projective space? This is of interest in connection with the coordinatization of complemented modular lattices and in particular of continuous geometries, since if the corresponding projective space is irreducible, it determines a unique division ring (unless it is a projective plane).

The results on projective spaces, in particular the characterization Theorem 7 , suggest similar results concerning generalized affine spaces. Other definitions of projective coordinate spaces suggest themselves. In particular one may omit the condition that the coordinate functions $d(t)$ vanish except for a finite number of values of the argument $t$.

Another question is, to what extent does the principle of duality hold in projective spaces of infinite dimension, and in particular in the spaces $P S(\alpha, D)$ ? The complements or duals of points are hyperplanes, and it is natural to consider the dual projective space whose elements (points) are these hyperplanes with the natural definition of "collinearity" or linear dependence. It is interesting to note that this dual space is not dually isomorphic to the original space in the infinite-dimensional case, and in fact may even be of different dimensionality (cardinal number $\alpha$ ).

\section{BIBLIOGRAPHY}

1. Garrett Birkhoff, Lattice theory, Amer. Math. Soc. Colloquium Publications, vol. 25, 1940.

2. - Combinatorial relations in projective geometries, Ann. of Math. (2) vol. 36 (1935) pp. 743-748.

3. - Subdirect unions in universal algebra, Bull. Amer. Math. Soc. vol. 50 (1944) pp. 764-768.

4. R. P. Dilworth, Note on complemented modular lattices, Bull. Amer. Math. Soc. vol. 46 (1940) pp. 74-76.

5. - Ideals in Birkhoff lattices, Trans. Amer. Math. Soc. vol. 49 (1941) pp. 325-353.

6. R. P. Dilworth and M. Hall, The imbedding problem for modular lattices, Ann. of Math. (2) vol. 45 (1944) pp. 450-456. 
7. Orrin Frink, Jr., Representations of Boolean algebras, Bull. Amer. Math. Soc. vol. 47 (1941) pp. 755-756.

8. Saul Gorn, Homomorphisms and modular functionals, Trans. Amer. Math. Soc. vol. 51 (1942) pp. 103-116.

9. M. Hall, Projective planes, Trans. Amer. Math. Soc. vol. 54 (1943) pp. 229-277.

10. Karl Menger, New foundations of projective and affine geometry, Ann. of Math. (2) vol. 37 (1936) pp. 456-482.

11. M. H. Stone, The theory of representations for Boolean algebras, Trans. Amer. Math. Soc. vol. 40 (1936) pp. 37-111.

12. - Topological representations of distributive lattices and Brouwerian logics, Casopis pro Péstovánı Matematiky a Fysiky vol. 67 (1937) pp. 1-25.

13. Oswald Veblen and W. H. Young, Projective geometry, 2 vols., Boston, 1910.

14. John von Neumann, Lectures on continuous geometries, vols. 1-3, Princeton, 1936-37.

15. Henry Wallman, Lattices and topological spaces, Ann. of Math. (2) vol. 39 (1938) pp. 112-126.

The Pennsyluania State College, State College, Pa. 